\title{
Targeted development of specific biomarkers of endometrial stromal cell differentiation using bioinformatics: the IFITM1 model
}

\author{
Carlos E Parra-Herran, Liping Yuan, Marisa R Nucci and Bradley J Quade \\ Department of Pathology, Women's and Perinatal Pathology Division, Brigham and Women's Hospital, \\ Boston, MA, USA
}

\begin{abstract}
When classifying cellular uterine mesenchymal neoplasms, histological distinction of endometrial stromal from smooth muscle neoplasms can be difficult. The only widely established marker of endometrial stromal differentiation, CD10, has marginal specificity. We took a bioinformatics approach to identify more specific markers of endometrial stromal differentiation by searching the Human Protein Atlas, a public database of protein expression profiles. After screening the database using different methods, interferon-induced transmembrane protein 1 (IFITM1) was selected for further analysis. Immunohistochemistry for IFITM1 was performed using tissue sections from the selected cases of proliferative endometrium (22), secretory endometrium (6), inactive endometrium (19), adenomyosis (10), conventional leiomyoma (11), cellular leiomyoma (16), endometrial stromal nodule (2), low-grade endometrial stromal sarcoma (16), high-grade endometrial stromal sarcoma (2) and undifferentiated uterine sarcoma (2). Stained slides were scored in terms of intensity and distribution. Normal endometrial samples uniformly showed diffuse and strong IFITM1 staining. Endometrial stromal neoplasms, particularly low-grade endometrial stromal sarcoma, showed higher IFITM1 expression compared with smooth muscle neoplasms $(P<0.0001)$. IFITM1 immunohistochemistry has high sensitivity and specificity, particularly in the distinction between low-grade endometrial stromal sarcoma and leiomyoma ( 81.2 and $86.7 \%$, respectively). Our results indicate that IFITM1 is a sensitive and specific marker of endometrial stromal differentiation across the spectrum from proliferative endometrium to metastatic stromal sarcoma. IFITM1 is a potential valuable addition to immunohistochemical panels used in the diagnosis of cellular mesenchymal uterine tumors. Further studies with larger number of cases are necessary to corroborate this impression and determine the utility of IFITM1 in routine practice. This study is a clear example of how bioinformatics, particularly tools for mining genomic and proteomic databases, can enhance and accelerate biomarker development in diagnostic pathology.
\end{abstract}

Modern Pathology (2014) 27, 569-579; doi:10.1038/modpathol.2013.123; published online 27 September 2013

Keywords: endometrial stroma; endometrial stromal neoplasms; IFITM1

Recognition of endometrial stroma, either normal or neoplastic, is relevant in many diagnostic situations. One of the most important tasks, given its prognostic and therapeutic implications, is the distinction between endometrial stromal tumors and smooth muscle tumors. Although histomorphology is sufficient to make this distinction in most cases, differentiating low-grade endometrial stromal

Correspondence: Dr BJ Quade, MD, PhD, Department of Pathology, Harvard Medical School or Women's and Perinatal Pathology Division, Brigham and Women's Hospital, 75 Francis Street, Boston, MA 02115, USA.

E-mail: bquade@partners.org

Received 16 January 2013; revised 13 May 2013; accepted 14 May 2013; published online 27 September 2013 sarcoma from leiomyoma and cellular leiomyoma can be particularly challenging.

Immunohistochemistry is the most common ancillary technique applied to distinguish between smooth muscle and endometrial stromal differentiation. Frequently used markers include CD10, h-caldesmon, desmin and smooth muscle actin. $\mathrm{H}$-caldesmon is the most specific and sensitive indicator of smooth muscle differentiation. ${ }^{1,2}$ Other markers of smooth muscle, such as desmin and smooth muscle actin, have lesser value due to their variable expression in endometrial stromal tumors.

CD10 (common acute lymphoblastic leukemia antigen) is widely used as the best marker for endometrial stromal differentiation in clinical practice. It is uniformly expressed in normal endometrial 
Table 1 Gene products with high/strong expression in endometrial stromal cells and low/absent expression in endometrial glands and smooth muscle, identified by the bioinformatic queries in the Human Protein Atlas database

\section{Name}

Symbol

R3H domain and coiled-coil containing 1

Kinesin family member 12

Low-density lipoprotein receptor-related protein-associated protein 1

Interferon-induced transmembrane protein 1

R3HCC1

KIF12

LRPAP 1

IFITM1

stromal cells and is highly sensitive in the diagnosis of endometrial stromal sarcoma. ${ }^{3-5}$ Expression of CD10 is, however, not specific of endometrial stromal cells and can be seen in many other cell populations, including hematopoietic cells, renal tubular epithelium and smooth muscle cells among others. Moreover, $20-30 \%$ of benign and malignant smooth muscle tumors can express CD10, which reduces its specificity. ${ }^{6-10}$

Limitations of the current immunohistochemical markers prompted us to search for better indicators of endometrial stromal differentiation. We took a bioinformatics approach to identify and test new and potentially more specific markers of endometrial stromal differentiation by searching the Human Protein Atlas, a public database containing immunohistochemistry-based protein expression profiles interrogating a large number of human tissues, cancers and cell lines using tissue microarrays. This approach allowed us to identify interferon-induced transmembrane protein 1 (IFITM1) as a potentially useful marker. In this study, we analyze the expression of IFITM1 by immunohistochemistry in normal endometrium and myometrium, as well as in endometrial stromal and smooth muscle tumors.

\section{Materials and methods}

\section{Biomarker Search}

The bioinformatic search for new biomarkers for endometrial stromal cell differentiation began by downloading the 'normal tissue data' file from the Human Protein Atlas project (available at http:// proteinatlas.org/about/download). This $3.4 \mathrm{MB}$ comma-separated values-formatted downloaded copy contained 790019 rows. Each row has data fields for the Ensembl gene name, tissue name, cell type, expression level and staining reliability (as a qualitative score) for each observation. Expression level is annotated as a 'staining pattern' if the target protein was tested with only one antibody, or as an 'Annotation of Protein Expression' for instances in which $\geq 2$ antibodies directed towards the same protein target were tested. This file was imported into a data table we named 'Normal tissue' in Microsoft Access 2010. Using Transact Structured Query Language, three queries were constructed with wild card parameters to identify genes products that had either (1) 'high' or 'strong' staining in endometrial stroma, (2) 'low' or 'very low' staining in endometrial glands and (3) 'low' or 'very low' staining in smooth muscle. Specifically, the queries were:

(1) 'SELECT Normal_tissue.Gene FROM Normal tissue WHERE (((Normal_tissue.Tissue) Like 'uter*') AND ((Normal_tissue.[Cell type]) Like '*stroma*') AND ((Normal_tissue.Level) = 'high' Or (Normal_ tissue.Level) = 'strong')) ORDER BY Normal_tissue. Gene;'.

(2) 'SELECT Normal_tissue.Gene FROM Normal tissue WHERE (((Normal_tissue.Tissue) Like 'uter*') AND ((Normal_tissue.[Cell type]) Like '*gland*') AND ((Normal_tissue.Level) Like '*low*')) ORDER BY Normal tissue.Gene;'.

(3) 'SELECT Normal_tissue.Gene, Normal_tissue. Tissue, Normal_tissue.[Cell type], Normal_tissue. Level FROM Normal_tissue WHERE (((Normal tissue.Tissue) Like 'smooth muscle') AND ((Normal_ tissue.Level) = 'low' Or Normal_tissue.Level) = 'weak' Or (Normal_tissue.Level) = 'none' Or (Normal_tissue.Level) = 'negative')) OR ((Normal_tissue.[Cell type]) Like 'smooth muscle*') AND $($ Normal_tissue.Level) $=$ 'low' Or (Normal_tissue. Level) = 'weak' Or (Normal_tissue.Level) = 'none' Or Normal_tissue.Level)='negative')) ORDER BY Normal_tissue.Gene;'.

The asterisks (*) denote use of the wild card operator; for example, this operator allowed the database engine to match multiple, similar text strings like 'uterus' and 'uterine'. Multiple selections of the same protein were possible if the matching staining results were based on $>1$ antibody. The first query identified 842 genes, the second query 1146 genes and the third query 8186 genes. Two subsequent queries were designed to find the genes that were common to queries 1 and 2 as well as 1 and 3 . As a result, we identified 10 unique genes common to queries 1 and 2, 119 genes common to queries 1 and 3 and 4 genes common to all three queries (see Table 1). Using the Protein Atlas website, the full staining scores as well as representative images of antibody staining of tissue microarray(s) were reviewed by two investigators (CPH and BJQ) to verify each potential candidate and determine the staining level in non-scored adjacent myometrium and in other tissues containing smooth muscle. This visual review rejected all genes but IFITM1 for further investigation.

As a second method of bioinformatics screening, we performed a visual inspection of the Access data table, sorting the spreadsheet by tissue type (level 1), cell type (level 2) and level of staining (level 3). After sorting, cases with 'high (for 'Annotation of Protein Expression') or 'strong' (for cases with target proteins tested by a single antibody) staining in endometrial stroma were selected. This selection included 50 possible target proteins. Similar to the first method, we proceeded to review full staining 
Table 2 Average IFITM1 IHC expression in the mesenchyme of normal endometrium, endometrial stromal neoplasms and smooth muscle neoplasms ${ }^{\text {a }}$

\begin{tabular}{|c|c|c|c|c|}
\hline Category & Diagnosis & $\mathrm{N}$ & $\begin{array}{l}\text { Average IFITM1 } \\
\text { intensity }\end{array}$ & $\begin{array}{l}\text { Average IFITM1 } \\
\text { distribution }\end{array}$ \\
\hline \multirow[t]{4}{*}{ Normal endometrium } & Proliferative endometrium & 22 & 2.91 & 2.86 \\
\hline & Secretory endometrium & 6 & 2.83 & 2.67 \\
\hline & Inactive endometrium & 19 & 3 & 2.79 \\
\hline & Adenomyosis & 10 & 2.9 & 2.7 \\
\hline \multirow{6}{*}{$\begin{array}{l}\text { Endometrial stromal } \\
\text { neoplasms }\end{array}$} & Endometrial stromal tumor of any type & 22 & 2.36 & 2.09 \\
\hline & Endometrial stromal sarcoma, low grade & 13 & 2.54 & 2.31 \\
\hline & $\begin{array}{l}\text { Metastatic endometrial stromal sarcoma, low } \\
\text { grade }\end{array}$ & 3 & 2 & 2 \\
\hline & Endometrial stromal nodule & 2 & 2 & 2 \\
\hline & Endometrial stromal sarcoma, high grade & 2 & 2 & 1 \\
\hline & Undifferentiated uterine sarcoma & 2 & 3 & 2 \\
\hline \multirow[t]{2}{*}{ Smooth muscle neoplasms } & Leiomyoma, usual type & 11 & 0.73 & 0.36 \\
\hline & Cellular leiomyoma & 16 & 0.88 & 0.63 \\
\hline
\end{tabular}

${ }^{\mathrm{a}}$ In normal endometrium cases, expression refers to endometrial stroma. See text for statistical comparisons.

scores and microarray staining images for each candidate protein (Human Protein Atlas web site), assessing staining in myometrium, smooth muscle and endometrial glands. Only two proteins showed absent expression in such tissues: IFITM1 and mannose receptor $\mathrm{C}$ type 2 . Therefore, we selected IFITM1 as the best potential marker for further analysis.

\section{Case Selection and Review}

We selected cases of proliferative endometrium (22 cases), secretory endometrium (6), inactive endometrium (19 cases) and adenomyosis (10 cases) and retrieved representative formalin-fixed, paraffinembedded tissue from our pathology department archives. We similarly selected cases of leiomyoma (11 cases), cellular leiomyoma (16 cases), endometrial stromal nodule (2 cases), low-grade endometrial stromal sarcoma (16 cases), high-grade endometrial stromal sarcoma (2 cases) and undifferentiated uterine sarcoma (2 cases).

Available hematoxylin and eosin-stained slides were reviewed, and the diagnosis was confirmed in each case. Endometrial stromal neoplasms were classified as endometrial stromal nodule if they had sharp circumscription and were composed of a uniform population of cells with round-to-ovoid nuclei resembling proliferative-phase endometrial stroma; blood vessels were evenly spaced and uniform in caliber. Tumors were classified as lowgrade endometrial stromal sarcoma if they had the same cytological features of an endometrial stromal nodule but showed evident myometrial infiltration or invasion of lymphatic or vascular spaces. Tumors were classified as high-grade endometrial stromal sarcoma as recently described if the tumor contained morphologically high-grade areas composed of round/epithelioid cells arranged in nests with a delicate stromal capillary network, the tumor cells showed large nuclei with irregular nuclear contours and there was significant mitotic activity $(>10$ mitoses/10 HPF). ${ }^{11}$ The diagnosis of undifferentiated uterine sarcoma was made in tumors with evident and diffuse nuclear atypia and pleomorphism without morphological evidence of endometrial stromal differentiation. The number of cases and distribution by final diagnosis are represented in Table 2.

\section{Immunohistochemistry}

Primary polyclonal antibody to IFITM1 (HPA004810) was purchased from Sigma-Aldrich (St Louis, MO). The immunogen used to prepare this antibody consisted of the following recombinant, 36-residue protein fragment: MHKEEHEVAVLGAPPSTILPRSTVINIHSETSVPDH. This polypeptide corresponds to the first (aminoterminal) 36 amino-acid residues of the human IFITM1. Immunogen was injected into rabbits; primary antibody was affinity purified. Immunohistochemistry protocol used $4-\mu \mathrm{m}$-thick tissue sections on microscope slides that were deparaffinized, and endogenous peroxidase activity was blocked by $1 \%$ hydrogen peroxide in methanol. Epitope retrieval was performed by heating under pressure in $10 \mathrm{mM}$ sodium citrate, $0.05 \%$ Tween 20, pH 6.0 using a Decloaking Chamber (Biocare Medical, Concord, CA) set to $120^{\circ} \mathrm{C}$. The conditions for the primary antibody (1:400, overnight at $4{ }^{\circ} \mathrm{C}$ ) were empirically determined by testing serial dilutions in phosphatebuffered saline on slides of normal endomyometrium. The negative control was omission of the primary antibody. Primary antibody binding was detected using the two-step Envision + anti-rabbit HRP-labeled polymer (K4003, Dako, Carpinteria, CA) and DAB-Plus substrate kit (002010, Invitrogen, Grand Island, NY) following the manufacturers' directions. 

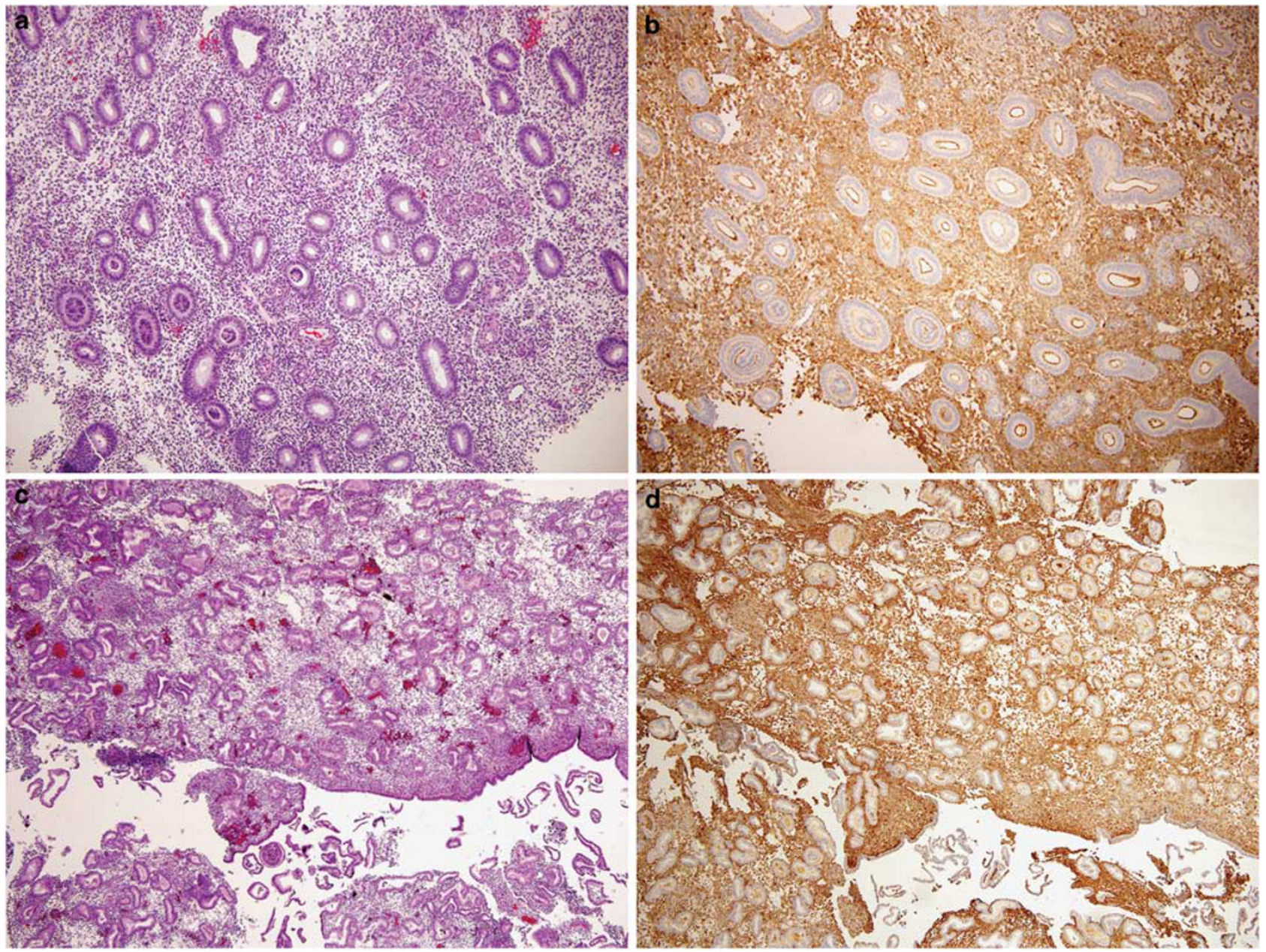

Figure 1 IFITM1 expression in proliferative (a and $\mathbf{b}$ ) and secretory phase (c and $\mathbf{d}$ ) endometria (hematoxylin and eosin- $-\mathbf{a}$ and $\mathbf{c}$; IHC—-b and d. Original magnification $\times 40$ ).

\section{Interpretation and Statistical Analysis}

Immunohistochemical stains were independently reviewed and interpreted by three pathologists (CPH, MRN and BJQ). Scoring was performed using a semi-quantitative score for intensity and distribution of staining. Intensity was graded as 0 (absent), 1 (mild), 2 (moderate) and 3 (strong). Distribution was graded as 0 (absent, $\leq 5 \%$ of cells), 1 (patchy, $6-25 \%$ of cells), 2 (multifocal, $26-75 \%$ of cells) and 3 (diffuse, $\geq 75 \%$ of cells). Scores of intensity and distribution were registered by every pathologist, and a final consensus score was determined in each case (the final score is the score given by the majority of reviewers, either three out of three or two out of three). Of note, complete disagreement (all three pathologists giving different scores) did not occur in any of the cases analyzed. Once consensus scores for intensity and distribution were obtained, a final 'combined' IFITM1 score was calculated (sum average of intensity and distribution scores).

Scores for intensity and distribution of staining of neoplastic cases (endometrial stromal neoplasms and smooth muscle neoplasms) were compared using the Wilcoxon-Mann-Whitney Test. A difference was considered statistically significant if the two-sided $P$ value was $<0.05$. In addition, immunohistochemical results in the neoplastic cases were grouped as positive (intensity and distribution scores 2 or 3 , combined score $>1.5$ ) or negative (intensity and distribution scores 0 or 1 , combined score $\leq 1.5$ ). Positive and negative cases for IFITM1 were distributed by diagnostic category, and sensitivity, specificity, positive and negative likelihood ratios were calculated.

This study was reviewed and approved by the Partners Human Research Committee.

\section{Results}

Normal endometrium samples consistently showed strong and diffuse IFITM1 staining in endometrial stromal cells (Table 2). Expression was high in both proliferative and secretory phase endometria (Figure 1), as well as in inactive endometrium (Figure 2). In contrast, endometrial glands and 

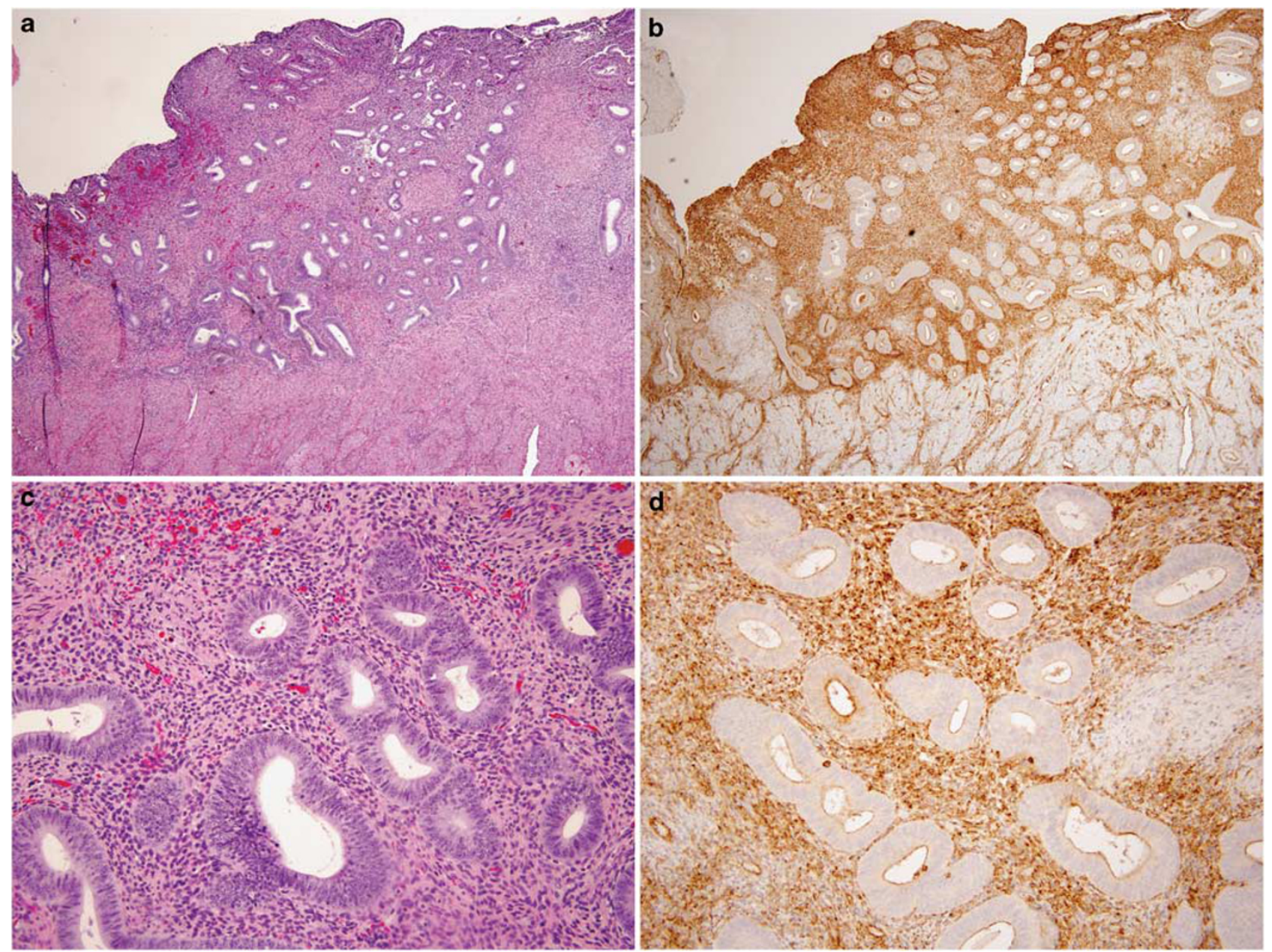

Figure 2 IFITM1 expression in inactive endometrium. Strong and diffuse staining in endometrial stromal cells. Endothelial cells also stain for IFITM1. Endometrial glands and myometrium are negative (hematoxylin and eosin-a and c; IHC-b and d. Original magnification $\times 40-\mathbf{a}$ and $\mathbf{b} ; \times 200-\mathbf{c}$ and $\mathbf{d}$ ).

myometrium were negative (Figures 1 and 2). Additionally, staining of the gland lumina, lymphocytes and endothelial cells was consistently observed.

IFITM1 staining results for individual neoplastic cases are reported in Table 3. A total of 22 endometrial stromal tumors were analyzed. Most of them were either primary or metastatic low-grade endometrial stromal sarcoma (16 cases). The remaining cases represented endometrial stromal nodule, high-grade endometrial stromal sarcoma and undifferentiated uterine sarcoma. The endometrial stromal tumor group showed moderate-tostrong staining intensity (average score 2.36) and multifocal-to-diffuse staining distribution (average score 2.09, Figures 3 and 4). In contrast, IFITM1 staining in the 30 smooth muscle tumors analyzed was either absent or focal in most cases (average intensity score 0.73 for conventional leiomyoma, 0.88 for cellular leiomyoma and 0.74 for the combination; average distribution score 0.36 for conventional leiomyoma, 0.63 for cellular leiomyoma and 0.48 for the combination. See Figure 5). The difference in staining between endometrial stromal and smooth muscle neoplasms was statistically significant $(P<0.0001)$ for both staining intensity and distribution. Such difference was also significant between subgroups (low-grade endometrial stromal sarcoma versus all leiomyomas $P<0.0001$, low-grade endometrial stromal sarcoma versus conventional leiomyoma $P<0.0001$, low-grade endometrial stromal sarcoma versus cellular leiomyoma $P=0.003$ )

Other endometrial stromal tumor types also were tested in limited numbers due to their rarity (Tables 2 and 3). One endometrial stromal nodule showed only mild and patchy staining for IFITM1, whereas the other showed strong and diffuse expression. Our two high-grade endometrial stromal sarcomas had moderate and patchy staining, and our two undifferentiated uterine sarcoma cases showed strong and multifocal staining.

Table 4 shows calculated sensitivity, specificity and likelihood ratios. When classifying the immunohistochemical results as positive or negative for IFITM1 expression, sensitivity and specificity in 
Table 3 IFITM1 IHC staining scores (intensity and distribution) in endometrial stromal and smooth muscle neoplasms ${ }^{\mathrm{a}}$

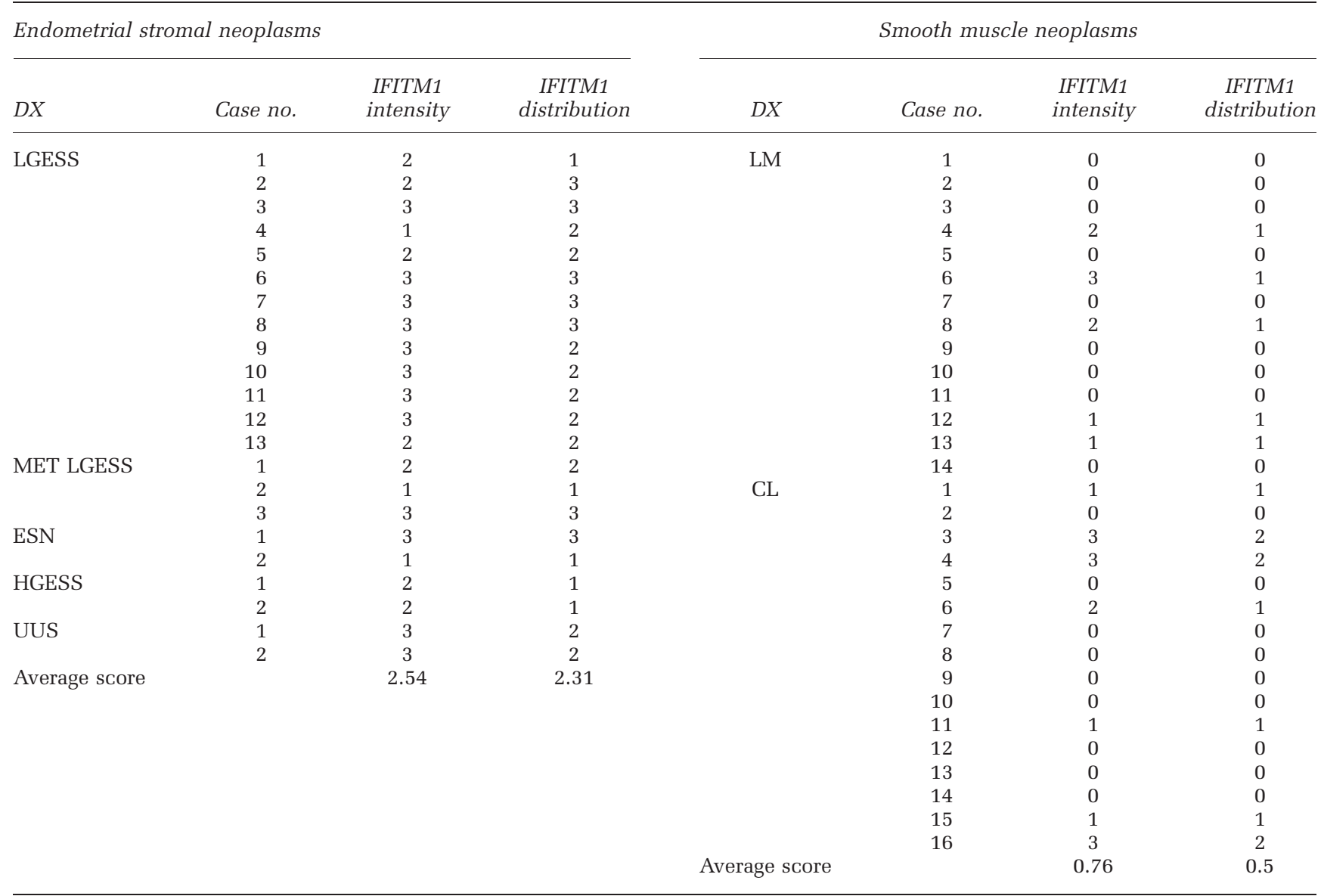

${ }^{\mathrm{a}}$ See text for statistical comparisons.

the diagnosis of endometrial stromal tumors and lowgrade endometrial stromal sarcoma are high. Using a combined immunohistochemistry score, sensitivity and specificity were highest in the distinction between low-grade endometrial stromal sarcoma and smooth muscle tumors (81.2 and $86.7 \%$, respectively). Likewise, immunohistochemistry for IFITM1 has satisfactory positive and negative likelihood ratios in the diagnosis of endometrial stromal tumors and low-grade endometrial stromal sarcoma versus smooth muscle tumors and cellular leiomyoma.

\section{Discussion}

In our study, we found that expression of IFITM1 in normal endometrium is consistently high in endometrial stroma, independent of the hormonal status (active versus inactive endometrium) and menstrual cycle phase (proliferative versus secretory). In the samples of normal tissue examined, endometrial glands and myometrium were negative for IFITM1 expression. In situ hybridization studies have shown IFITM1 mRNA levels to be mainly expressed in endometrial stromal cells in mice, ${ }^{12}$ which is consistent with our immunohistochemistry results.
Endometrial stromal neoplasms showed consistent IFITM1 expression by immunohistochemistry. Expression measured by intensity and distribution of staining was significantly higher in endometrial stromal tumors and low-grade endometrial stromal sarcoma compared with smooth muscle tumors and cellular leiomyomas. Likewise, when classifying cases as positive or negative for IFITM1 expression, sensitivity and specificity were high. In addition, both a positive result (defined as moderate/strong and multifocal/diffuse staining) and a negative result (defined as absent/weak and absent/patchy staining) had a high likelihood ratio in the diagnosis of endometrial stromal neoplasms. Differences in staining, as well as sensitivity and specificity, were higher when separating low-grade endometrial stromal sarcomas from other endometrial stromal tumors. Our data suggest that IFITM1 has higher specificity than CD10 in the distinction between endometrial stromal and smooth muscle neoplasms (particularly low-grade endometrial stromal sarcoma).

We included cases of endometrial stromal nodule, high-grade endometrial stromal sarcoma and undifferentiated uterine sarcoma in our series to evaluate their IFITM1 expression. Unfortunately, the number of cases in these diagnostic categories is low, 

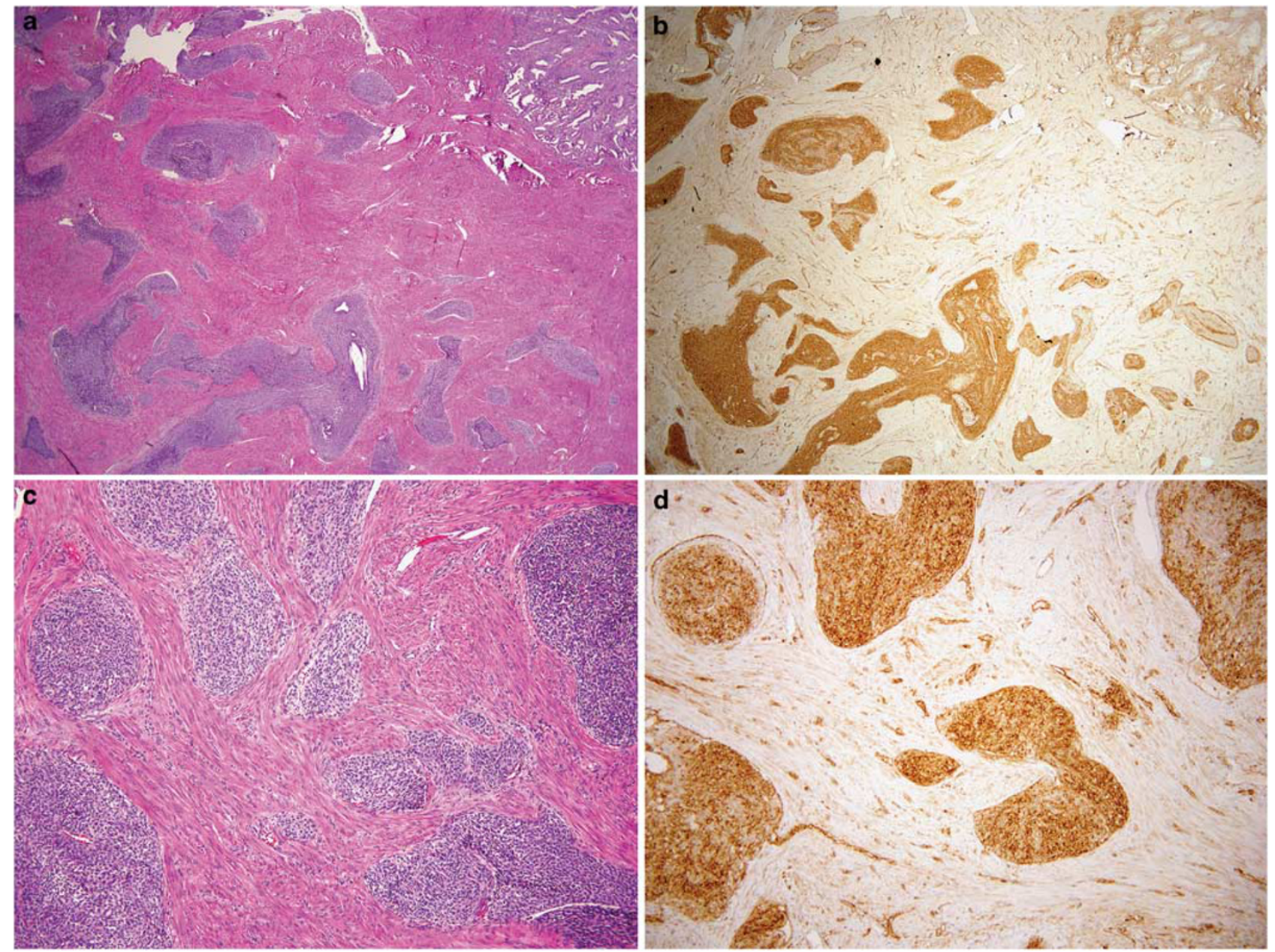

Figure 3 IFITM1 expression in low-grade endometrial stromal sarcoma. Strong cytoplasmic staining is noted in both normal and neoplastic endometrial stromal cells (hematoxylin and eosin-a and $\mathbf{c}$; IHC $-\mathbf{b}$ and $\mathbf{d}$. Original magnification $\times 20-\mathbf{a}$ and $\mathbf{b}, \times 100-\mathbf{c}$ and $\mathbf{d})$.

limiting our analysis. Interestingly, one endometrial stromal nodule and both high-grade endometrial stromal sarcomas had only patchy staining (score 1) and were considered negative by using the combined immunohistochemistry score classification described. Loss of staining for CD10 is typically observed in high-grade endometrial stromal sarcomas. Our results could represent a similar phenomenon for IFITM1. On the other hand, both cases of undifferentiated uterine sarcoma showed strong and multifocal staining. Undifferentiated uterine sarcoma is a rare diagnosis, and the biology of these neoplasms is poorly understood. It is still unclear whether undifferentiated uterine sarcoma arises in endometrial stroma or other mesenchymal precursor. Considering that IFITM1 is, based on our results, a specific marker of endometrial stromal differentiation, its expression in our cases of undifferentiated uterine sarcoma would suggest of an endometrial stromal origin.

Despite significant differences in intensity and distribution of IFITM1 expression, and clear separation of IFITM1 immunohistochemical results between endometrial stromal and smooth muscle tumors, the latter category was not entirely negative. Moderate/strong staining was observed in $23.3 \%$ $(7 / 30)$ and multifocal/diffuse staining in 10\% (3/30) of cases. These findings may impact the value of IFITM1 in the diagnosis of low-grade endometrial stromal sarcoma. Combining intensity and distribution of staining in the interpretation, however, greatly improves specificity while maintaining optimal sensitivity ( 86.7 and $81.2 \%$ in our study, respectively). Addition of smooth muscle markers will also improve the diagnostic efficacy in challenging cases. In this regard, we suggest an immunohistochemical panel that includes markers of both endometrial stromal (IFITM1 and CD10) and smooth muscle (h-caldesmon, desmin and smooth muscle actin) differentiation.

Although expression of IFITM1 in the uterus appears to be specific for endometrial stromal cells, the protein is present in a variety of other normal tissues as well. Expression of IFITM1 has been detected by immunohistochemistry using antibody HPA004810 in lymphoid non-germinal center cells, 

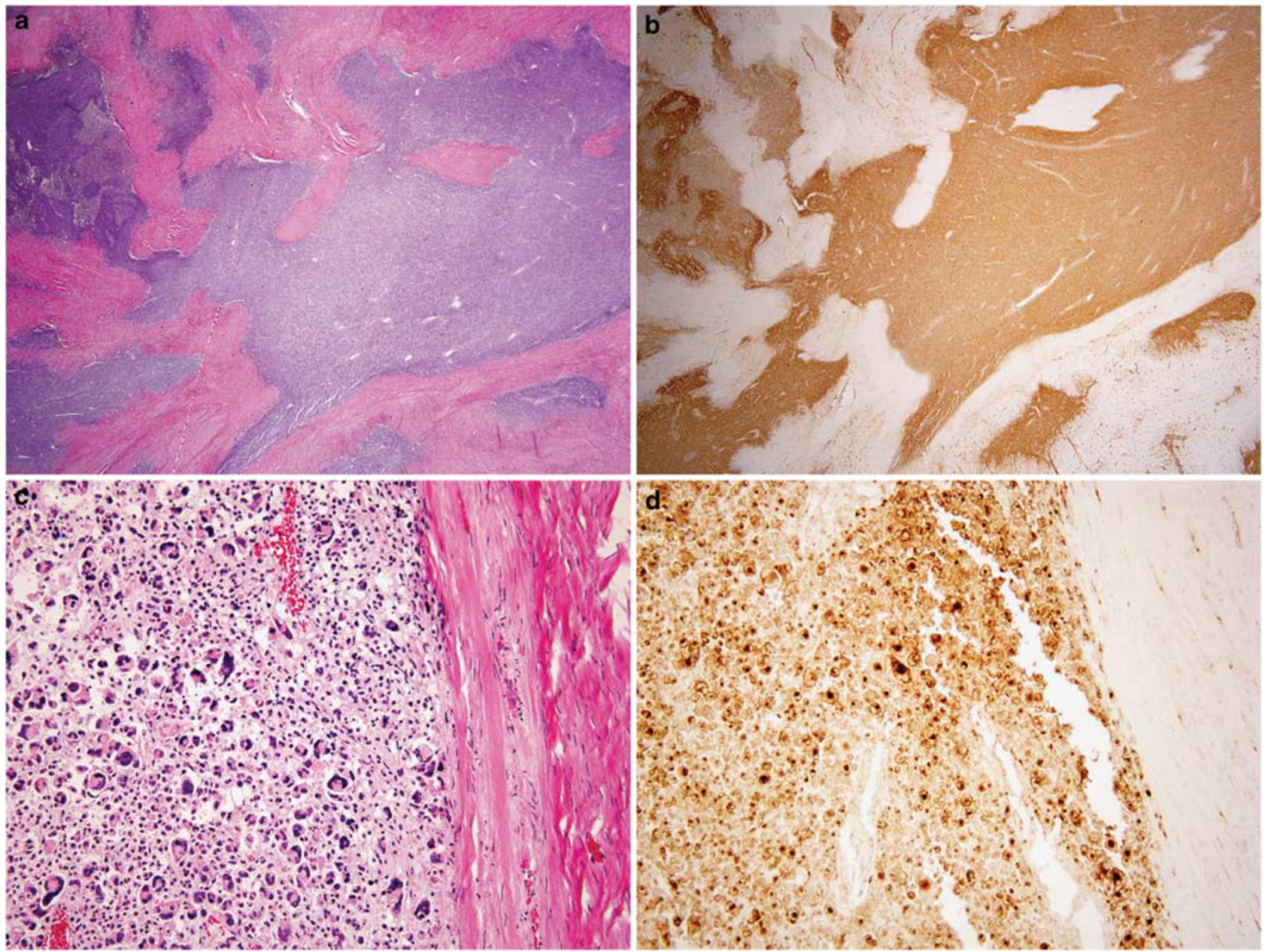

Figure 4 IFITM1 expression in low-grade endometrial stromal sarcoma (a and $\mathbf{b}$ ) and undifferentiated uterine sarcoma (c and $\mathbf{d})$. Notice the strong and diffuse staining in the former and the strong nuclear and less intense cytoplasmic staining in the latter (hematoxylin and eosin- $\mathbf{a}$ and $\mathbf{c}$; IHC $-\mathbf{b}$ and $\mathbf{d}$. Original magnification $\times 20-\mathbf{a}$ and $\mathbf{b}, \times 200-\mathbf{c}$ and $\mathbf{d}$ ).

ovarian stroma (strong cytoplasmic immunoreactivity), endothelial cells (moderate cytoplasmic positivity), epididymal and colonic epithelium (luminal membranous immunoreactivity) and squamous and respiratory epithelium (basal layer immunoreactivity) http://www.proteinatlas.org/ENSG00000185885. Among these tissues, expression levels by normal endothelium and ovarian stroma represent a potential pitfall in the diagnosis of endometrial stromal neoplasms. This expression profile should be taken into consideration, and careful correlation with the histomorphology is necessary.

IFITM1 (also known as 9-27, Leu-13 and CD225) was first characterized as part of a gene family highly inducible by type I and II interferons. ${ }^{13,14}$ Although IFITM1 is constitutively expressed in many cells, ${ }^{15,16}$ de novo synthesis of IFITM1 can also be induced by $\alpha$ - or $\gamma$-interferon. ${ }^{14,17}$ Most of the anti-proliferative and growth-inhibitory effects of IFN- $\gamma$ are secondary to IFITM1 induction. In addition, IFITM1 has a key role in innate cellular immunity. ${ }^{15,18,19}$
Role of IFITM1 in the human female genital tract tissues is less understood. Some studies suggest a role in uterine growth and embryo implantation. In mice, uterine IFITM1 expression is part of the Wnt/ $\beta$-catenin signaling pathway, which appears to be crucial in hormone-mediated uterine growth and implantation. ${ }^{12,20,21}$ Furthermore, IFITM1 expression in murine uteri varies during the oestrus cycle, peaking during the late proestrus, oestrus and metoestrus phases (corresponding to the follicular phase and peri-ovulatory period of the menstrual cycle). ${ }^{12}$ Such expression also appears to correlate with levels of estrogen and gonadotropins in the mouse; however, we did not observe such cyclical expression in the normal human endometria. In addition, IFITM1 expression, measured by mRNA levels, is high in endometrial stromal cells compared with myometrial and glandular endometrial cells. ${ }^{12}$ Finally, IFITM1 appears to have a role in gonadal embryological development. The aminoterminal extracellular domain of the IFITM1 protein (which corresponds to the immunogen for 

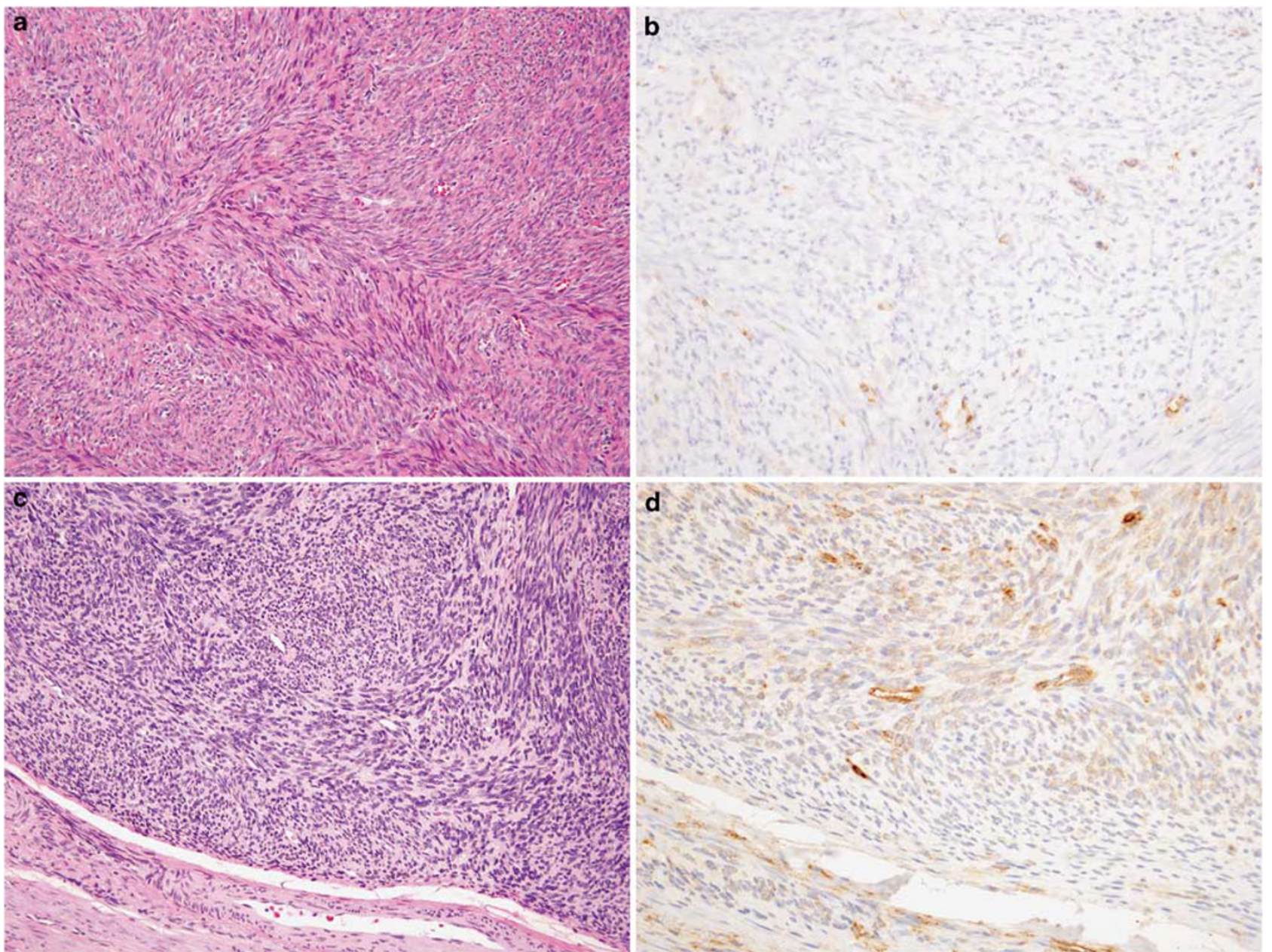

Figure 5 IFITM1 expression in leiomyoma (a and $\mathbf{b}$ ) and cellular leiomyoma (c and $\mathbf{d})$. Notice that staining is patchy and weak in smooth muscle cells (hematoxylin and eosin-a and $\mathbf{c}$; IHC $-\mathbf{b}$ and $\mathbf{d}$. Original magnification $\times 200$ ).

preparation of the antibody used herein) mediates the migration of primordial germ cells from the epiblastic mesoderm into the endoderm by repelling primordial germ cells from the allantoic bud. ${ }^{22}$

With the accelerated discovery of new genes and proteins, finding useful biomarkers for diagnostic, prognostic and therapeutic purposes may be challenging. It is also particularly difficult to integrate the use of new molecules in clinical practice using optimal, standardized and reproducible techniques. Genomic and proteomic databases integrate relevant information of currently characterized molecules, facilitating the search for new biomarkers. ${ }^{23,24}$ Among them, the Human Protein Atlas database contains a comprehensive, antibody-based database of protein expression and localization profiles of multiple human normal and tumoral tissues. ${ }^{25,26}$ The atlas also serves as a source of internally generated, validated antibodies. Data mining of proteomic databases like the Human Protein Atlas is an emerging source for the discovery and validation of biomarker candidates for several other tissue types and organ systems. ${ }^{27-31}$ In addition, exploration of proteomic data can potentially identify specific biomarkers of disease not identified by other methods, such as gene expression profiling. Indeed, a recent study describing gene expression signatures of endometrial stromal sarcomas and leiomyosarcomas did not identify IFITM1 as significantly differentially expressed in these two malignancies. ${ }^{32}$

The use of immunohistochemistry by the Human Protein Atlas was particularly desirable for our study, because it is a commonly used technique in routine pathology practice. In addition, the Atlas also includes digital tissue microarray immunohistochemical images distributed across the Internet, which allowed us to narrow our biomarker search by examining visually the marker expression in target tissues without the time and expense of physical experimentation in our laboratory before candidate selection. In fact, the interval from downloading the Human Protein Atlas data file to titering the candidate antibody preparation was approximately 2 weeks.

Of note, in addition to being distributed commercially, antibodies found in the Human Protein Atlas are extensively validated. This validation 
Table 4 Sensitivity, specificity, positive and negative likelihood ratios of IFITM1 IHC (intensity, distribution and combined scores) in the distinction between EST and SMT

\begin{tabular}{|c|c|c|c|c|c|c|}
\hline \multicolumn{3}{|l|}{ IFITM1 intensity } & \multirow[t]{2}{*}{ SEN (\%) } & \multirow[t]{2}{*}{$S P E(\%)$} & \multirow[t]{2}{*}{ POS LR (95\% CI) } & \multirow[t]{2}{*}{ NEG LR $(95 \%$ CI $)$} \\
\hline IHC & EST & SMT & & & & \\
\hline+ & 19 & 7 & 86.4 & 76.7 & $3.7(1.89-7.23)$ & $0.18(0.06-0.52)$ \\
\hline- & 3 & 23 & & & & \\
\hline IHC & LGESS & SMT & & & & \\
\hline+ & 14 & 7 & 87.5 & 76.7 & $3.75(1.91-7.36)$ & $0.16(0.04-0.61)$ \\
\hline- & 2 & 23 & & & & \\
\hline IHC & LGESS & CL & & & & \\
\hline+ & 14 & 4 & 87.5 & 75 & $3.5(1.47-8.34)$ & $0.17(0.04-0.63)$ \\
\hline- & 2 & 12 & & & & \\
\hline IFITM1 distribution & & & $\operatorname{SEN}(\%)$ & SPE $(\%)$ & POS LR (95\% CI) & NEG LR $(95 \%$ CI) \\
\hline IHC & EST & SMT & & & & \\
\hline+ & 17 & 3 & 77.3 & 90 & $7.73(2.58-23)$ & $0.25(0.12-0.55)$ \\
\hline- & 5 & 27 & & & & \\
\hline IHC & LGESS & SMT & & & & \\
\hline+ & 14 & 3 & 87.5 & 90 & $8.75(2.94-26)$ & $0.14(0.04-0.51)$ \\
\hline- & 2 & 27 & & & & \\
\hline IHC & LGESS & CL & & & & \\
\hline+ & 14 & 3 & 87.5 & 81.2 & $4.67(1.65-13)$ & $0.15(0.04-0.57)$ \\
\hline- & 2 & 13 & & & & \\
\hline IFITM1 combined & & & SEN (\%) & SPE $(\%)$ & POS LR (95\% CI) & NEG LR $(95 \% C I)$ \\
\hline IHC & EST & SMT & & & & \\
\hline+ & 16 & 4 & 72.7 & 86.7 & $5.45(2.11-14)$ & $0.31(0.16-0.63)$ \\
\hline- & 6 & 26 & & & & \\
\hline IHC & LGESS & SMT & & & & \\
\hline+ & 13 & 4 & 81.2 & 86.7 & $6.09(2.38-16)$ & $0.22(0.08-0.61)$ \\
\hline- & 3 & 26 & & & & \\
\hline IHC & LGESS & CL & & & & \\
\hline+ & 13 & 3 & 81.2 & 81.2 & $4.33(1.52-12)$ & $0.23(0.08-0.66)$ \\
\hline- & 3 & 13 & & & & \\
\hline
\end{tabular}

CI, confidence interval; CL, cellular leiomyoma; EST, endometrial stromal tumors; IFITM1, interferon-induced transmembrane protein 1; IHC, immunohistochemitry; LGESS, low-grade endometrial stromal sarcoma; NEG LR, negative likelihood ratio; POS LR, positive likelihood ratio; SEN, sensitivity; SMT, smooth muscle tumors; SPE, specificity.

encompasses protein-based assays (western blot analysis, immunohistochemistry and immunofluorescent-based confocal microscopy). ${ }^{33,34}$ Granted, the staining profile for IFITM1 in the Human Protein Atlas has not been fully validated. The atlas includes information on two antibodies for IFITM1: HPA004810 (the antibody used in this study, see Materials and methods) and SC66827 (Santa Cruz Biotechnology). The latter is a monoclonal antibody raised against the full-length recombinant protein. We believe that the data available on this second antibody validate our results on the polyclonal antibody tested herein by virtue of having a similar staining pattern (http:// www.proteinatlas.org/ENSG00000185885). Thus, it is quite reasonable to predict that our results with HPA004810 would be corroborated by an independent antibody preparation.

In summary, IFITM1 is a highly specific marker of endometrial stromal differentiation across the spectrum from proliferative endometrium to metastatic endometrial stromal sarcoma. Given its high specificity and sensitivity, IFITM1 appears to be superior to CD10 in the diagnosis of endometrial stromal tumors. IFITM1 appears to be a potential valuable addition to immunohistochemical panels used in the diagnosis of cellular uterine mesenchymal tumors. Studies including different antibodies and more tissue samples are needed to further determine the usefulness of IFITM1 in this and other clinical scenarios. Finally, this study is a clear example of how bioinformatics can enhance and accelerate biomarker development for diagnostic pathology.

\section{Disclosure/conflict of interest}

The authors declare no conflict of interest.

\section{References}

1 Nucci MR, O’Connell JT, Huettner PC, et al. $\mathrm{H}$-caldesmon expression effectively distinguishes endometrial stromal tumors from uterine smooth muscle tumors. Am J Surg Pathol 2001;25:455-463. 
2 Rush DS, Tan J, Baergen RN, et al. H-caldesmon, a novel smooth muscle-specific antibody, distinguishes between cellular leiomyoma and endometrial stromal sarcoma. Am J Surg Pathol 2001;25:253-258.

3 Chu PG, Arber DA, Weiss LM, et al. Utility of CD10 in distinguishing between endometrial stromal sarcoma and uterine smooth muscle tumors: an immunohistochemical comparison of 34 cases. Mod Pathol 2001;14: 465-471.

4 McCluggage WG, Sumathi VP, Maxwell P. CD10 is a sensitive and diagnostically useful immunohistochemical marker of normal endometrial stroma and endometrial stromal neoplasms. Histopathology 2001; 39:273-278.

5 Toki T, Shimizu M, Takagi Y, et al. CD10 is a marker for normal and neoplastic endometrial stromal cells. Int J Gynecol Pathol 2001;21:41-47.

6 Abeler VM, Nenodovic M. Diagnostic immunohistochemistry in uterine sarcomas: a study of 397 cases. Int J Gynecol Pathol 2011;30:236-243.

7 Oliva E, Young R, Amin M, et al. An immunohistochemical analysis of endometrial stromal and smooth muscle tumors of the uterus. Am J Surg Pathol 2002;26:403-412.

8 D’Angelo E, Spagnoli LG, Prat J. Comparative clinicopathologic and immunohistochemical analysis of uterine sarcomas diagnosed using the World Health Organization classification system. Hum Pathol 2009;40:1571-1585.

9 Zhu XQ, Shi YF, Cheng XD, et al. Immunohistochemical markers in differential diagnosis of endometrial stromal sarcoma and cellular leiomyoma. Gynecol Oncol 2004;92:71-79.

10 Koivisto-Korander R, Butzow R, Koivisto AM, et al. Immunohistochemical studies on uterine carcinosarcoma, leiomyosarcoma and endometrial stromal sarcoma: expression and prognostic importance of ten different markers. Tumor Biol 2011;32:451-459.

11 Lee CH, Ali RH, Rouzbahman M, et al. Cyclin D1 as a diagnostic immunomarker for endometrial stromal sarcoma with YWHAE-FAM22 rearrangement. Am J Surg Pathol 2012;36:1562-1570.

12 Park HJ, Kuk IS, Kim JH, et al. Characterization of mouse Interferon-induced transmembrane protein-1 gene expression in the mouse uterus during oestrus cycle and pregnancy. Reprod Fertil Dev 2011;23:798-808.

13 Lewin AR, Reid LF, McMahon M, et al. Molecular analysis of a human interferon-inducible gene family. Eur J Biochem 1991;199:417-423.

14 Reid LE, Brasnett AH, Gilbert CS, et al. A single DNA response element can confer inducibility by both $\alpha$ and $\gamma$ - interferons. Proc Natl Acad Sci 1989;86:840-844.

15 Huang IC, Bailey CC, Weyer JL, et al. Distinct patterns of IFITM-mediated restriction of filoviruses, SARS, coronavirus and influenza A virus. PLoS Pathogens 2011;7:1-13.

16 Friedman RL, Manly SP, McMahon M, et al. Transcriptional and posttranscriptional regulation of Interferoninduced expression in human cells. Cell 1984;38: $745-755$.
17 Yang G, Xu Y, Chen X, et al. IFITM1 plays an essential role in the antiproliferative action of interferon- $\gamma$. Oncogene 2007;26:584-603.

$18 \mathrm{Lu}$ J, Pan Q, Rong L, et al. The IFITM1 proteins inhibit HIV-1 infection. J Virol 2011;85:2126-2137.

19 Brass AL, Huang IC, Benita Y, et al. IFITM proteins mediate the innate immune response to influenza A H1N1 virus, West Nile virus and Dengue virus. Cell 2009;139:1243-1254.

20 Hou X, Tan Y, Li M, et al. Canonical Wnt signaling is critical to estrogen-mediated uterine growth. Mol Endocrinol 2004;18:3035-3049.

21 Xie H, Tranguch S, Jia X, et al. Inactivation of nuclear Wnt- $\beta$ catenin signaling limits blastocyst competency for implantation. Development 2008;135:717-727.

22 Tanaka SS, Yamaguchi YL, Lickert H, et al. IFITM/mil/ fragilis family proteins IFITM1 and IFITM3 play distinct roles in mouse primordial germ cell moning and repulsion. Dev Cell 2005;9:745-756.

23 Li J, Roebuck P, Grunewald S, et al. SurvNet: a web server for identifying network-based biomarkers that most correlate with patient survival data. Nucleic Acids Res 2012;40:W123-W126.

24 Fagerberg L, Strömberg S, El-Obeid A, et al. Large-scale protein profiling in human cell lines using antibodybased proteomics. J Proteome Res 2011;10:4066-4075.

25 Pontén F, Schwenk JM, Asplund A. The Human Protein Atlas as a proteomic resource for biomarker discovery. J Intern Med 2011;270:428-446.

26 Uhlén M, Björling E, Agaton C, et al. A human protein atlas for normal and cancer tissues based on antibody proteomics. Mol Cell Proteomics 2005;4:1920-1932.

27 Haggarth L, Hagglof C, Jaraj SJ, et al. Diagnostic biomarkers of prostate cancer. Scand J Urol Nephrol 2011;45:60-67.

28 Magnusson K, de Wit M, Brennan DJ, et al. SATB2 in combination with cytokeratin 20 identified over 95\% of all colorectal carcinoma. Am J Surg Pathol 2011;35:937-948.

29 Ehlen A, Nodin B, Rexhepaj E, et al. RBM3-regulated genes promote DNA integrity and affect clinical outcome in epithelial ovarian cancer. Transl Oncol 2011;4:212-221.

30 Edlund K, Lindskog C, Saito A, et al. CD99 is a novel prognostic stromal marker in non-small cell lung cancer. Int J Cancer 2012;131:2264-2273.

31 Lindskog C, Korsgren O, Ponten F, et al. Novel pancreatic beta cell-specific proteins: antibody-based proteomics for identification of new biomarker candidates. J Proteomics 2012;75:2611-2620.

32 Davidson B, Abeler VM, Hellesylt E, et al. Gene expression signatures differentiate uterine endometrial stromal sarcoma from leiomyosarcoma. Gynecol Oncol 2013;128:349-355.

33 Pontén F, Jirström K, Uhlen M. The Human Protein Atlas-a tool for pathology. J Pathol 2008;216:387-393.

34 Asplund A, Edqvist PH, Schwenk JM, et al. Antibodies for profiling the human proteome-The Human Protein Atlas as a resource for cancer research. Proteomics 2012;12:2067-2077. 